\title{
Antibiotic use at dental implant placement
}

\section{Abstracted from}

\section{Esposito M, Grusovin MG, Worthington HV.}

Interventions for replacing missing teeth: antibiotics at dental implant placement to prevent complications. Cochrane Database Syst Rev 2013; 7: CD004152. doi: 10.1002/14651858. CD004152.pub4. Review. PubMed PMID: 23904048.

Address for correspondence: Luisa Fernandez Mauleffinch, Managing Editor, Cochrane Oral Health Group, School of Dentistry, The University of Manchester, JR Moore Building, Oxford Road, Manchester, M13 9PL, UK.

E-mail: luisa.fernandez@manchester.ac.uk

Question: Is there a beneficial or harmful effect from the use of systemic antibiotic prophylaxis for dental implant placement versus no administration or use of a placebo? And which type, dose and duration is the most effective?

Data sources Cochrane Oral Health Group's Trial Register, Cochrane Central Register of Controlled Trials (CENTRAL), MEDLINE via OVID and EMBASE via OVID. Databases were searched with no language or date restrictions.

Study selection Two authors independently reviewed the titles and the abstracts for inclusion. Disagreements were resolved by discussion. If needed, a third author was consulted. Included were randomised clinical trials with a follow-up of at least three months which evaluated the use of prophylactic antibiotic compared to no antibiotic or a placebo and examined different antibiotics of different doses and durations in patients undergoing dental implant placement. The outcomes were implant failure (considered as implant mobility, removal of implant due to bone loss or infection) and prosthesis failure (prosthesis could not be placed).

Data extraction and synthesis Standard Cochrane methodology procedures were followed. Risk of bias was completed independently and in duplicate by two review authors. Results were expressed as risk ratios (RRs) using a random-effects model for dichotomous outcomes with $95 \%$ confidence intervals $(\mathrm{Cl})$. The statistical unit was the participant and not the prosthesis or implant. Heterogeneity including both clinical and methodological factors was investigated.

Results Six randomised clinical trials with 1162 participants were identified for the review. Three trials compared $2 \mathrm{~g}$ of preoperative amoxicillin versus placebo (927 participants). One trial compared $3 \mathrm{~g}$ of preoperative amoxicillin versus placebo (55 participants). Another trial compared $1 \mathrm{~g}$ of preoperative amoxicillin plus $500 \mathrm{mg}$ four times a day for two days versus no antibiotic ( 80 participants). An additional trial compared four groups: (1) $2 \mathrm{~g}$ of preoperative amoxicillin; (2) 2 $\mathrm{g}$ of preoperative amoxicillin plus $1 \mathrm{~g}$ twice a day for seven days; (3)

This paper is based on a Cochrane Review published in the Cochrane Library 2013, issue 7 (see www.thecochranelibrary.com for information). Cochrane Reviews are regularly updated as new evidence emerges and in response to feedback, and the Cochrane Library should be consulted for the most recent version of the review.
$1 \mathrm{~g}$ of postoperative amoxicillin twice a day for seven days and (4) no antibiotics (100 participants). The overall body of the evidence was considered moderate.

The meta-analysis of the six trials showed a statistically significant higher number of implant failures in the group not receiving antibiotics, $\mathrm{RR}=0.33(95 \% \mathrm{Cl} ; 0.16$ to 0.67$) \mathrm{P}=0.0002$.

The number needed to treat for one additional benefit outcome (NNTB) to prevent one person having an implant failure is 25 (95\% $\mathrm{Cl} ; 14$ to 100 ) based on an implant failure of $6 \%$ in participants not receiving antibiotics.

There was borderline statistical significance for prosthesis failures $(\mathrm{RR}=0.44(95 \% \mathrm{Cl} ; 0.19$ to 1.00$)$ with no statistically significant differences for infections or adverse events. No conclusive information for the different durations of antibiotics could be determined Conclusions There is statistically significant evidence suggesting that a single dose of $2 \mathrm{~g}$ or $3 \mathrm{~g}$ of amoxicillin given orally is beneficial in reducing dental implant failure in ordinary conditions. No significant adverse events were reported. It is still unknown whether post-operative antibiotics are beneficial and which antibiotic is more effective.

\section{Commentary}

Antibiotics are very important and needed drugs. Since their discovery the benefits cannot be calculated for their role in halting and treating minor to severe bacterial infections.

However, for the last few years we have been dealing with overuse, misuse and abuse of antibiotics. Many bacteria are no longer susceptible to antibiotics, commonly used drugs are less effective and patients have more allergic reactions.

As healthcare providers we play an important role in judging the appropriate use of antibiotics. The important issue is to consider which procedures alter the risk-benefit ratio in favour of using antibiotic prophylaxis in healthy patients.

Risk factors are associated with an increased risk of infection, which may be systemic, or local factors.

The review analysed the beneficial use of prophylactic antibiotics for dental implant placement under ordinary conditions, along with the dose and time frame needed, the type of antibiotic and the possible side effects.

As a Cochrane produced review, the authors followed the correct methodology for systematic reviews and appropriately searched and selected randomised clinical trials to answer the proposed question.

Six articles were selected and analysed, three of which were assessed with a high risk of bias and three studies were assessed 
with a low risk (two of the clinical trials were done by the principal author of the systematic review).

The evidence from the meta-analysis shows statistically significant results for the use of preoperative antibiotics, specifically amoxicillin, however the clinical significance is debatable.

There are many variables and cofounders to be taken into consideration for early implant failure even in healthy patients.

The number needed to treat for one additional benefit outcome (NNTB) calculated in the review provides additional information about the effect size that providers may find useful in clinical decision making. Single digit values of number needed to treat (NNT) usually represent a useful difference when comparing one intervention to another. However in this case an NNT of 25 (range of $14-100)$ seems quite large for the decision to use preoperative antibiotic, which indicates that we need to treat 24 patients with antibiotic in order to prevent one implant failure.

The results showed no benefit in preventing post-operative infection and no side effects were reported.

The results seem convincing, however providers need to balance the risk and benefits in order to preoperatively prescribe $2-3 \mathrm{~g}$ of amoxicillin to patients prior to implant placement to prevent early implant failure.

\section{Analia Veitz-Keenan ${ }^{a}$ and James R Keenan ${ }^{b}$ ${ }^{a}$ NYU College of Dentistry, New York, USA} ${ }^{b}$ Private Practice, New York, USA

Evidence-Based Dentistry (2015) 16, 50-51. doi:10.1038/sj.ebd.6401096 